- Angiogenesis is driven not only by hypoxia, but also by proinflammatory mediators produced by immune and stromal cells

- Many pathways downstream of these proinflammatory stimuli contribute to various cellular processes involved in angiogenesis

- Targeting proangiogenic pathways to inhibit neovascularization has been successfully exploited in several cancers, and may also prove beneficial in the treatment of chronic inflammatory diseases

Disclosure of Interest: None declared

DOI: 10.1136/annrheumdis-2017-eular.7153

\section{SP0049 DNA AGGREGATES AS ALARMINS}

D. Pisetsky. Medicine, Duke University Medical Center, Durham, North Carolina, United States

DNA is a large polymeric molecule that displays powerful immunological activities and operates in the context of multi-component aggregates to alarm the immune system and stimulate innate immunity. Although DNA has important roles in normal host defense, DNA can serve as an autoantigen and autoimmunogen by itself or in association with other immunologically active nuclear molecules. In the setting of systemic lupus erythematosus (SLE), DNA is a key target antigen; while antibodies to pure DNA double stranded DNA serve as important biomarkers, the relevant antigenic form of DNA during disease is the nucleosome in which DNA is bound to histones. Furthermore, nucleosomes can be components of microparticles which are small membrane-bound structures that are released from dead and dying cells. MPs can stimulate immune responses and serve as a nidus for immune complex formation. In normal immunity, DNA can interact with nucleic acid sensors in the cytoplasm of cells to stimulate responses including production of type 1 interferon. These sensors respond to DNA from intracellular organisms such as bacteria and viruses although damaged DNA and DNA from mitochondria can also interact with these receptors. While these sensors are intracellular, they can interact with extracellular DNA that is introduced or transfected into the cell; this translocation event occurs with DNA bound with other molecules. In another facet of host defense, DNA can be released from neutrophils during a process termed NETosis. A NET or neutrophil extracellular trap is a mesh-like structure comprised of DNA as well as granule proteins that have antibacterial activity. NETs can trap and kill bacteria. Thus, in its diverse immunological roles, DNA interacts with other molecules to form aggregates or sub-cellular structures that alarm the immune system, promote host defense or drive critical events in autoimmunity.

Disclosure of Interest: None declared

DOI: 10.1136/annrheumdis-2017-eular.7271

\section{THURSDAY, 15 JUNE 2017 \\ Bringing rheumatology research to the next level: addressing the main challenges of patient partnerships in research and health care service design}

\section{SP0050 ENSURING REPRESENTATIVENESS OF THE PATIENTS' PERSPECTIVES IN THE FINAL RESULTS GENERATED FROM CLINICAL RESEARCH - CHALLENGES FROM THE PERSPECTIVE OF RESEARCHERS}

A.-M. Orbai on behalf of GRAPPA-OMERACT Psoriatic Arthritis Core Set Working Group. Rheumatology, Johns Hopkins University School of Medicine, Baltimore, United States

Now more than ever, patients are increasingly taking part in shared healthcare decisions with their physicians. Information from clinical research, on which evidence-based medicine draws upon, ideally needs to make sense to patients and not only physicians.

Representativeness of the patients' perspectives in the final results of clinical research is therefore critical for shaping the content and quality of medical knowledge and for shared clinical decision-making. As a result there is increased emphasis and even requirement from organizations around the world to include patient research partners (PRPs) as equal members in the medical research team. PRP inclusion means participation in research question generation, study design, data analysis/interpretation, authorship and results dissemination. Several questions remain: 1) How can we maximize the impact of the perspective patients bring to the table? 2) How can we ensure we capture the patients' perspective accurately and carry it forward into the final product of research? We can maximize impact and reach by including the patients' perspective in research that defines the way we assess disease and disease targeted interventions. Randomized controlled trials (RCTs) and longitudinal observational studies (LOS) are currently our main source of clinical information regarding efficacy of disease targeted interventions. Outcomes assessed in RCTs and LOS, as well as the health measurement tools used to assess these outcomes need to include the patient perspective in order for the information generated from these studies to be valid and usable. A conceptual diagram to illustrate how the patients' perspective is critical in generating health information for patient and physician shared decision making is represented in the Figure.

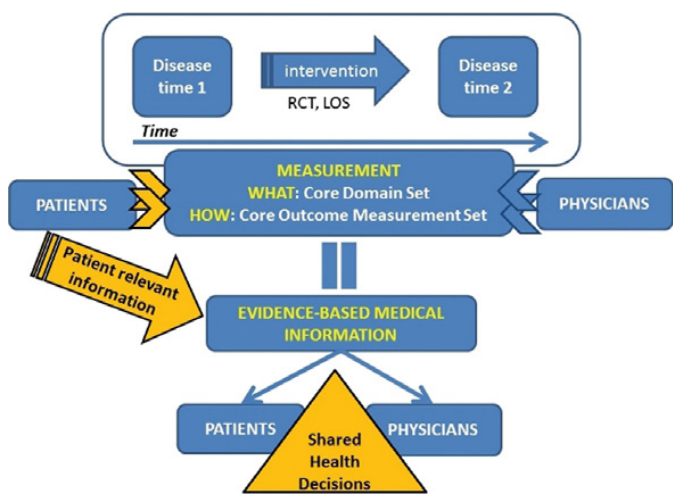

Case study: the updated Psoriatic Arthritis (PsA) Core Domain Set patient domains. A core domain set is the minimum set of outcomes that need to be assessed to evaluate the effect of disease targeted interventions. The first PsA core domain set was developed in 2006 by physicians and methodologists. The core set update study identified clinical trial outcomes important for patients as a group and for physicians as a group and reconciled both perspectives to recommend an updated PsA core domain set that represents the perspective of both patients and physicians. Five PRPs were members of the working group and contributed during all stages of the project. The perspective of patients was included as follows: a) domain generation through qualitative data collection and analysis from international focus groups; b) first domain prioritization exercise in electronic surveys with patients and physicians; c) a face to face nominal group technique meeting with 12 patients and 12 physicians to prioritize domains; d) second domain prioritization exercise through electronic surveys with patients and physicians; e) discussion and voting at the 2016 Outcome Measures in Rheumatology conference. During the process of achieving consensus we observed the following dynamic for patient prioritized domains (percentages represent proportions of patients ranking each domain important in the first survey and then the second survey): 1) Pain was rated important by $76 \%$ of patients in the first survey and $82 \%$ in the second survey and remained a core domain; 2) Fatigue $78 \%$ then $71 \%$ and became core domain; 3) Physical function $72 \%$ then $80 \%$ and remained a core domain; 4) Participation (daily activities and employment/work) was important to $72-76 \%$ of patients in the first survey and to $78 \%$ in the second survey and became a middle circle domain (important, not required); 5) Emotional well-being $60 \%$ then $57 \%$ and was placed in the middle circle; 6 ) Independence $82 \%$ then $63 \%$ and placed on the research agenda. In each of these situations the trend in the patient's vote as a group followed the physician's vote with one exception: the domain participation.

Summary of challenges and solutions for ensuring representativeness of the patients' perspective in the final results: Patients may change their views and align with physicians during the course of a research project. PRPs can help increase awareness of the patients' perspective throughout all stages of the research process. Special attention should be given to: 1) use of methods that adequately capture the patient perspective in the initial stages of a research project, and 2) allowing a robust patient perspective to take shape from patients as a group, before the consensus process with additional stakeholders begins. In addition, supporting patients with adequate information and materials and involving PRPs in their development will maximize understanding of the task for patients and their full participation.

Disclosure of Interest: None declared

DOI: 10.1136/annrheumdis-2017-eular.7245

\section{SP0051 BECOMING A PATIENT RESEARCH PARTNER IN THE FIELD OF RHEUMATOLOGY. MY EXPECTATIONS AND THE CHALLENGES OF BEING EDUCATED AND TAKEN SERIOUSLY}

E.F. Mateus ${ }^{1,2,3}$. ${ }^{1}$ LIGA PORTUGUESA CONTRA AS DOENÇAS REUMÁTICAS, Lisbon, Portugal; ' 2 Patient Research Partner, EULAR, Zurich, Switzerland; ${ }^{3}$ ENP, EUPATI Portugal, Lisbon, Portugal

Patients often feel that their experience of illness is not valued nor understood by others. However, there has been a growing recognition of the importance of the patient perspective and involvement in research, improving its methodology and outcomes. The European Medicines Agency has been involving patients' and consumers' representatives on their work since 2005. The "European League Against Rheumatism recommendations for the inclusion of patient representatives in scientific projects" have been published in the "Annals of the rheumatic diseases" in 2011.

I was diagnosed with Juvenile Idiopathic Arthritis when I was 5 years old, back in 1977. Therefore, I have 40 years of experience of living with a Rheumatic and Musculoskeletal Disease (RMD), but no recollection of what my life was like "before" and "after" the diagnosis. I have learned to live and cope with my RMD, with a sense of being different from my peers. This sense of "uniqueness" was 
only disrupted when I realised that my experience of illness was quite common to other persons with a RMD. I can still remember my fascination, in a Patient Reported Outcomes workshop, by discovering that our experience as patients had a bigger relevance that the one we usually conceive.

Then, I felt the need of understanding the research process and specific jargon, so I could relate them with my knowledge on living with a RMD. Otherwise, any insight on the patient perspective would be "lost in translation", due to an inability to establish a correspondence between the scientific terminology and mindset regarding the disease, and the experience of illness and sickness by the individuals. Training opportunities were also recognised as desirable by the above mentioned EULAR recommendations, to increase expertise and understanding of research methods and to promote the patients' self-confidence on their contribution to research.

The Patient Research Partners (PRP) training by EULAR, in 2013, was the first step towards a better understanding of what could be the role of patients and on how to provide a meaningful input from the patients' perspective into research processes. It was followed in 2014 by the training provided by European Patients' Academy on Therapeutic Innovation (EUPATI) for Patient Expert on the Medicines Research and Development Process. This expert-level training was organized in a mixture of independent e-learning coursework and face-to-face training events over a 14-month period. The syllabus involved six modules: Discovery of Medicines \& Planning of Medicine Development, Non-Clinical Testing and Pharmaceutical Development, Exploratory and Confirmatory Clinical Development, Clinical Trials, Regulatory Affairs, Medicinal Product Safety, Pharmacovigilance and Pharmacoepidemiology, HTA principles and practices. Additionally, in 2016, I have attended the 1st EULAR course on Health Economics in Rheumatology.

In the meantime, my background as an anthropologist led me to become interested in Medical Anthropology. Between 2007-2015 my academic research (MA Phil. and PhD) was oriented towards a specialization in Anthropology of Health, with a special interest in Anthropology of Pharmaceuticals.

Based on my personal experience, on this lecture I will focus on the challenges of the role of the PRP trying to fill the gap between the mindsets and practices of different stakeholders.

Navigating through different meanings of symptoms and treatments, the educated patient representative must act like a translator, decodifying the biologic impact of the disease and intervention over the experience of illness on the everyday aspects of living with a RMD. The biggest expectation and challenge might be to bring these aspects forward, as relevant for the other stakeholders, since they shape individual values and patient's preferences.

Although recognised as having a pivotal role, patient's involvement in research may be limited by tokenism or by ineffective patients' participation. Patients' involvement is now a requirement and an added value to any project. But, is the project team ready and willing to listen to patients? Are PRP duly involved in the project, or are they just expected to be recipients without any input of their perspectives into the development and implementation of the research?

The knowledge and education acquired to perform our task enables us to understand science enough to communicate the patient experience in a meaningful way, improving the research. Our added value is, undoubtedly, our experience with the disease, our understanding of the individual values and preferences shaped by the everyday aspects of living with a RMD. We should be taken more seriously, for the benefit of science and patients.

Disclosure of Interest: None declared

DOI: 10.1136/annrheumdis-2017-eular.7243

\section{SP0052 BUILDING PATIENT PARTNERSHIP IN HEALTH CARE SERVICE DESIGN AND DELIVERY}

\section{H. Lempp. Rheumatology, Kings College London, London, United Kingdom}

This paper will present details of the approach to patient and public involvement in health service delivery, health service research and health care education in England. The presentation will be based upon a Logical Framework with the following key elements: inputs, processes, outputs, and outcomes/impacts. Key barrier acting to minimise the impact of building patient partnerships will be discussed, illustrated by examples from our experience in the preparatory stages for our departmental strategy to formalise close Patient Partnership for our research portfolio: (i) establish honorary contracts for patients for the academic Institution and local Hospital Trust; (ii) include patients on the interview panels to appoint project researchers (iii) build in a separate funding within the overall project budget for the costs associated with patient partnership and (iv) manage patient expectations of rapid implementation of the results of research after the completion of projects. Patient Partnership is essential and feasible to make health care service and design relevant.

\section{References:}

[1] Otto AK, Novielli K, Morahan PS.(2006) Implementing the logic model for measuring the value of faculty affairs activities. Acad Med. 2006 Mar;81(3):280-5.

[2] Mockford C, Staniszewska S, Grifiths F, Herron-Marx S. The impact of patient and public involvement on UK NHS health care: a systematic review. International Journal for Quality in Health Care, 2012; 24:28-38.

Disclosure of Interest: None declared

DOI: 10.1136/annrheumdis-2017-eular.7123

\section{THURSDAY, 15 JUNE 2017 WIN \& HOT session}

\section{SP0053 HOT SESSION: VASCULITIS TREATMENT}

\section{R.A. Luqmani. University of Oxford, Oxford, United Kingdom}

The systemic vasculitides are characterized by inflammation of blood vessels, which if untreated, results in tissue or end organ damage or death. Chapel Hill nomenclature is widely used to define different forms of vasculitis according to the size of the predominantly affected vessels. Other forms of vasculitis are not defined by a predominant vessel size (e.g. Behcet's syndrome). Vasculitis associated with the presence of anti-neutrophil cytoplasm antibody (ANCA), termed AAV, is less common than giant cell arteritis (GCA), but considerable advances have been made in understanding the pathogenesis and evidence based treatment for AAV. AAV is divided into three major forms: granulomatosis with polyangiitis (GPA) (Wegener's granulomatosis), microscopic polyangiitis (MPA) and eosinophilic granulomatosis with polyangiitis (EGPA) (Churg-Strauss syndrome). ANCA are implicated in their pathogenesis but not all patients with AAV are ANCA positive. We will review recent EULAR guidelines on therapy for AAV, based on careful structured clinical evaluation of patients, with stratification according to severity. Cyclophosphamide or rituximab (plus glucocorticoid) is used for severe disease, followed by maintenance with azathioprine (AZA) or methotrexate (MTX), and reducing doses of glucocorticoids; or maintenance rituximab. Additional plasmapheresis is indicated for very severe disease; by contrast for less severe disease, MTX or AZA or mycophenolate (plus glucocorticoids) can be used. The evidence for effectiveness is clear for MPA and GPA. A number of studies are underway to improve our use of these existing agents and to test newer, mechanism based treatments such as inhibition CTLA4lg or of the C5 complement pathway in GPA and MPA. For EGPA with severe manifestations, cyclophosphamide and glucocorticoids are recommended. A trial of mepolizumab (inhibitor of interleukin 5 , a potent driver of eosinophil production) in EGPA has recently been completed. IL-6 inhibition with tocilizumab is a significant advance over glucocorticoid monotherapy in treatment of GCA. Apremilast is effective in treating mucocutaneous manifestations of Behcet's syndrome. Relapse is a common feature of many forms of vasculitis and needs to be monitored by structured clinical evaluation. Monitoring of ANCA titres in AAV or the acute phase response in most forms of vasculitis can be misleading and should not serve as sole guide to therapy in the absence of clinical evidence of active disease. Early survival is over $94 \%$ of patients with most forms of vasculitis. Five year survival is $70-75 \%$ for AAV with current therapy. However, if the condition is more severe disease, especially with significant renal impairment, mortality is worse.

Vasculitis remains a challenge. Whilst mortality has dramatically reduced as a result of effective immunosuppression, relapse and chronic damage are significant problems for all forms of vasculitis. We need a better understanding of how to manage and limit the long term chronic effects of vasculitis and its therapy.

Disclosure of Interest: R. Luqmani Grant/research support from: Arthritis Research UK, GSK, MRC, UCSF/OIF, Canadian Institutes of Health Research, The Vasculitis Foundation; Consultant for: GSK, Medpace, Medlmmune, Roche DOI: 10.1136/annrheumdis-2017-eular.7162

\section{THURSDAY, 15 JUNE 2017 Treat-to-target in axSpA: reality or utopy?}

\section{SP0054 THE CONCEPT OF TREAT-TO-TARGET}

\section{J. Braun. Rheumazentrum Ruhrgebiet, 44649 Herne, Germany}

Many illnesses including most rheumatic diseases have substantial effects on well-being and quality of life, including deterioration of physical and mental function and a reduced life expectancy, since they can cause damage to organs and cells. If healing and regeneration cannot be achieved an impairment of organ function can be expected. In acute diseases this may occur rapidly over hours to days and weeks, while it often takes months to years in chronic diseases. However, if treatment is instituted early enough, organ damage may be prevented or diminished.

Critical for an optimal management of diseases with potentially severe outcomes is to determine the responsible thresholds for, for example, disease activity or to define the maximum level of a surrogate marker at which damage is unlikely to occur and, thus, will not be harmful in the long term. Although the optimal aim of therapy is cure, and appropriate therapy may even normalize life expectancy, many chronic diseases such as hypertension, diabetes, rheumatoid arthritis (RA) and ankylosing spondylitis (AS) have remained without curative therapies in the last decades - even though considerable progress has been made. Thus, a strategic therapeutic approach should aim for prevention of future damage, and maximal improvement of compromised organ function. Therefore, a clearly defined threshold of a validated measure that predicts future harm or no or minimal harm, is a target of critical importance for chronic diseases with potentially severe outcomes. Treat-to-target strategies having been developed to achieve this have widespread implications. They should be routinely followed - as long as the potential harm from treatment is carefully balanced against its benefit. Clearly, if inappropriately managed, the consequences of diabetes and hypertension in 